\title{
Planar near-field antenna measurement method based on symplectic relation and reaction concept
}

\author{
Yong-Heui Cho \\ School of Information and Communication Engineering \\ Mokwon University, Daejeon, 302-729, Republic of Korea
}

\begin{abstract}
Using symplectic relation and reaction concept, we propose a planar near-field antenna measurement method. A generalized probe compensation equation is deduced to obtain the probe correction formulation. To verify our approach, a reflector antenna with $1 x 2$ horn array is fabricated and measured in the near-field measurement facility. The near-field measurement results are compared with the physical optics (PO) simulation. The results of measurement and simulation agree very well near to the mainbeam.
\end{abstract}

Keywords: Communication system, antenna, measurement, symplectic relation, reaction concept.

\section{INTRODUCTION}

In a wireless communication system, antenna characteristics including bandwidth, antenna gain, and radiation patterns are very important to maintain the desired quality of service $(\mathrm{QoS})$. The communication antenna is mainly measured with far-field, Fresnel-zone, and near-field techniques [1]-[6]. The near-field antenna measurement is more effective than other measurement techniques. The near-field measurement facility has simple setup and requires less absorbing material than a far-field facility. However, near-field data are quite different from farfield radiation pattern and then these data in the near-field should be transformed into the far-field range in terms of the Fourier transform. Moreover, the near-field data contain the interaction of antenna under test (AUT) and measurement probe. The coupling effect of measurement probe should be removed to obtain precise far-field radiation pattern of AUT. This procedure is called as probe compensation. The probe compensation algorithm is significant to increase the accuracy of measurement [2]-[6]. The probe compensation is a little involved because of coupled integral equations [2], [3].

In this work, we will propose a generalized probe compensation equation based on symplectic relation and reaction concept to solve coupled integral equations effectively. In the first step, we introduce the Lorentz reciprocity theorem in terms of symplectic form and reaction. Then, the deduced probe compensation equation is applied to a planar measurement surface on which the electromagnetic fields can be expanded in plane-wave spectrum. The coupled integral equations are solved with the Fourier transform technique. Our proposed methodology is general yet analytic and can be

This is an excellent paper selected from the papers presented at ICCC 2009.

*Corresponding author. E-mail : yongheuicho@gmail.com

Manuscript received Mar. 29, 2010 ; accepted Jun. 15, 2010 extended to any measurement surface such as plane, circular cylinder, or sphere.

\section{GENERALIZED PROBE COMPENSATION EQUATION}

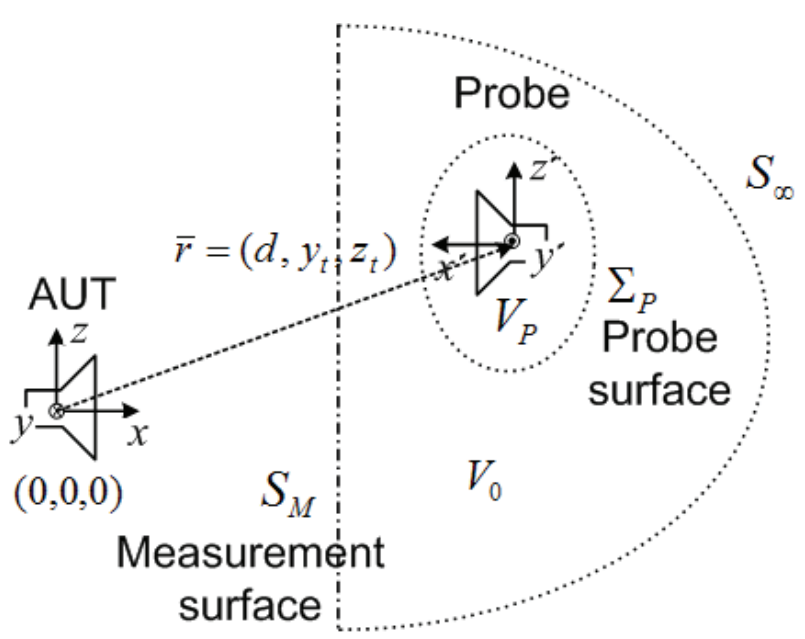

Fig. 1. Near-field antenna measurement setup

Although a generalized probe compensation equation is already proposed in [5], [6], we will rewrite the important procedures for the readers' convenience. The probe compensation is essential in order to get precise far-field pattern with near-field antenna measurement. This is because the measured near-field results are combined with AUT and probe characteristics.

To deduce a generalized probe compensation equation, we utilize symplectic relation [5] and reaction concept [7]. A symplectic vector space is usually defined to produce the dual 
or complementary space of an inner product space, which is widely used in mathematics and communication theory. The symplectic vector space has symplectic relation for its operator. The symplectic relation is very similar to subtraction in algebra, whereas the inner product is related to addition [5]. Then, the symplectic relation for self terms always results in zero and the inner product for them is zero or positive.

We use the Lorentz reciprocity theorem [3] to decouple the effects of AUT and probe interactions. In fact, the Lorentz reciprocity theorem has symplectic characteristics. Thus, it is reasonable to combine the Lorentz reciprocity theorem and symplectic relation. In electromagnetics, the Lorentz reciprocity theorem is represented in differential form as

$$
\begin{aligned}
& \bar{\nabla} \cdot\left(\bar{E}_{a} \times \bar{H}_{b}-\bar{E}_{b} \times \bar{H}_{a}\right) \\
& =\left(\bar{E}_{b} \cdot \bar{J}_{a}-\bar{H}_{b} \cdot \bar{M}_{a}\right)-\left(\bar{E}_{a} \cdot \bar{J}_{b}-\bar{H}_{a} \cdot \bar{M}_{b}\right)^{\prime}
\end{aligned}
$$

where subscripts, $a$ and $b$, imply that the electromagnetic fields are produced from the symbolized sources, $a$ and $b$. To represent (1) in compact form, we introduce the symplectic form and the Rumsey's reaction as

$$
\langle\langle a, b\rangle\rangle=\langle a, b\rangle-\langle b, a\rangle \text {, }
$$

where

$$
\begin{aligned}
& \langle\langle a, b\rangle\rangle=\oint_{s}\left(\bar{E}_{a} \times \bar{H}_{b}-\bar{E}_{b} \times \bar{H}_{a}\right) \cdot d \bar{a} \\
& \langle a, b\rangle=\int_{v}\left(\bar{E}_{b} \cdot \bar{J}_{a}-\bar{H}_{b} \cdot \bar{M}_{a}\right) d v .
\end{aligned}
$$

Note that $\langle a, b\rangle$ is a Rumsey's reaction [7] or a generalized power. When $a=b$, (3) is always zero, which shows that $\langle\langle a, b\rangle\rangle$ has symplectic relation. Similarly when $a=b,(4)$ is zero or positive due to electromagnetic radiation from the source $a$. From this observation, $\langle a, b\rangle$ is known as a generalized power.

Since the differential equation (1) is reformulated in terms of algebraic notations, we can easily apply our theory to near-field measurement setup illustrated in Fig. 1. In our following deduction, we ignore multiple reflections between AUT and probe. We only consider the radiated fields from AUT and probe, $a$ and $p$, and separately scattered fields from AUT and probe, $a s$ and $p s$. For example, the term $a s$ is equivalent source to represent the scattered field from AUT, when the incident field is from the source $p$ for probe field. Then, the reciprocity relation for setup of Fig. 1 is given by

$$
\begin{aligned}
& \langle\langle a+p s, p+a s\rangle\rangle_{\Sigma} \\
& =\langle a+p s, p+a s\rangle_{V}-\langle p+a s, a+p s\rangle_{V},
\end{aligned}
$$

where $\quad V=V_{0}+V_{P} \quad, \quad \Sigma=S_{M}+S_{\infty}, \quad$ and $\bar{M}_{a, p, a s, p s}=0$. Note that the volume $V$ only includes the sources, $p$ and $p s$. Manipulating (5) yields a generalized probe compensation equation as [5]

$$
\langle\langle a, p\rangle\rangle_{S_{M}}=2\langle p s, a s\rangle_{V_{P}}-\langle p, a\rangle_{V_{P}},
$$

where $\langle\langle a, p\rangle\rangle_{S_{M}}$ is a symplectic relation on the measurement surface $S_{M},\langle p, a\rangle_{V_{P}}$ is a generalized power for probe and AUT on the probe volume $V_{P}$, and $\langle p s, a s\rangle_{V_{P}}$ is very small compared to $\langle p, a\rangle_{V_{P}}$. When the source $p$ is fixed, $\langle p, a\rangle_{V_{P}}$ is only proportional to the incident electric field from $a$. Thus, $\langle p, a\rangle_{V_{P}}$ is analytically related to an open-circuit probe voltage function $P\left(\bar{r}_{0}\right)$ [3], [8] which can be directly measured at the end of measurement probe. The position vector $\bar{r}_{0}$ is defined as that from AUT to probe.

When $\left.\langle p, a\rangle_{V_{P}}>\right\rangle\langle p s, a s\rangle_{V_{P}}$, we obtain a simpler equation as

$$
\langle\langle a, p\rangle\rangle_{S_{M}} \approx-\langle p, a\rangle_{V_{P}}=P\left(\bar{r}_{0}\right) .
$$

Note that we obtain (7) based on small scattering approximation ( $p s<<1$ and $a s<<1$ ).

Using (7), we can predict the symplectic relation for AUT and probe. The measurement surface $S_{M}$ can be any surface such as plane, circular cylinder, or sphere.

In this work, (7) is applied to planar near-field antenna measurement. Utilizing the Weyl identity, the transverse electromagnetic fields radiated from AUT and probe in Fig. 1 are represented as [4]

$$
\begin{aligned}
& \bar{E}_{a}(\bar{r})=\frac{1}{2 \pi} \int \bar{t}_{10}\left(\bar{k}_{t}\right) e^{i \bar{k} \cdot \bar{r}} d \bar{k}_{t} \\
& \bar{E}_{p}\left(\bar{r}^{\prime}\right)=\frac{1}{2 \pi} \int \bar{s}_{20}\left(\bar{k}_{t}^{\prime}\right) e^{i \bar{k}^{\prime} \cdot \bar{r}^{\prime}} d \bar{k}_{t}^{\prime},
\end{aligned}
$$

where the magnetic fields can be formulated with the Maxwell's equations, the measurement surface $S_{M}$ in Fig. 1 is planar, AUT and probe are located at the origin and $\bar{r}=\left(d, y_{t}, z_{t}\right) \quad, \quad$ respectively, $\quad \bar{k}=k_{x} \hat{x}+\bar{k}_{t}$, $\bar{k}_{t}=k_{y} \hat{y}+k_{z} \hat{z} \quad, \quad \bar{r}^{\prime}=\left(d-x, y_{t}-y, z-z_{t}\right) \quad$, $\hat{x}^{\prime}=-\hat{x}, \hat{y}^{\prime}=-\hat{y}, \quad \hat{z}^{\prime}=\hat{z}$. It is noted that $\bar{t}_{10}\left(\bar{k}_{t}\right)$ and $\bar{s}_{20}\left(\bar{k}_{t}\right)$ are the inverse Fourier transform of $\bar{E}_{a}(\bar{r})$ and $\bar{E}_{p}(\bar{r})$, respectively. Inserting (8) and (9) into (7) yields a probe compensation equation as [4]

$$
\begin{aligned}
& \langle\langle a, p\rangle\rangle_{S_{M}}=P(\bar{r}) \\
& =\frac{2 k_{x}}{\omega \mu} \int \bar{t}_{10}\left(\bar{k}_{t}\right) \cdot \bar{s}_{20}\left(-\bar{k}_{t}\right) e^{i\left(k_{x} d+k_{y} y_{t}+k_{z} z_{t}\right)} d \bar{k}_{t} .
\end{aligned}
$$

When we measure $P(\bar{r})$ in near-field, we can determine $\bar{t}_{10}\left(\bar{k}_{t}\right) \cdot \bar{s}_{20}\left(-\bar{k}_{t}\right)$ in view of the Fourier transform, where $\bar{t}_{10}\left(\bar{k}_{t}\right)$ and $\bar{s}_{20}\left(\bar{k}_{t}\right)$ are related to the far-fields of AUT and 
probe, respectively. When $\bar{S}_{20}\left(\bar{k}_{t}\right)$ is known, we finally obtain the far-field of AUT.

\section{MEASUREMENTS}

To verify our formulations, (7) and (10), we measured a reflector antenna with $1 \times 2$ horn array shown in Fig. 2. The reflector antenna illustrated in Figs. 2 and 3 was designed with standard procedures. The diameter of a reflector is $700[\mathrm{~mm}]$ and the focus is determined with GO (Geometrical Optics) prediction. The feeder dimensions $\left(19.05 \times 16.5\left[\mathrm{~mm}^{2}\right]\right)$ are controlled to satisfy that the $10 \mathrm{~dB}$ beamwidth of a feeder covers the whole reflector. The measurement facility in Fig. 2 is located at the Electronics and Telecommunications Research Institute (ETRI), Daejeon, Korea. The near-field measurement facility has the dimensions as $3 \times 5 \times 2.5\left[\mathrm{~m}^{3}\right]$ and 8 inch pyramidal absorbers on all sides. The co-pole and cross-pole near-field patterns for the reflector antenna were obtained with the facility shown in Fig. 2, then we applied (10) to a near-field measurement and obtained the far-field of a reflector antenna by performing the probe compensation. The near-field measurement parameters are frequency $=15[\mathrm{GHz}]$, sampling period $=\lambda_{0} / 2$, scan range $=1[\mathrm{~m}] \times 1[\mathrm{~m}]$, AUT-probe distance $=10[\mathrm{~cm}]$, and probe $=$ WR-75 OERW (Open-Ended Rectangular Waveguide), where the AUT-probe distance is measured from the end of 1x2 horn array.

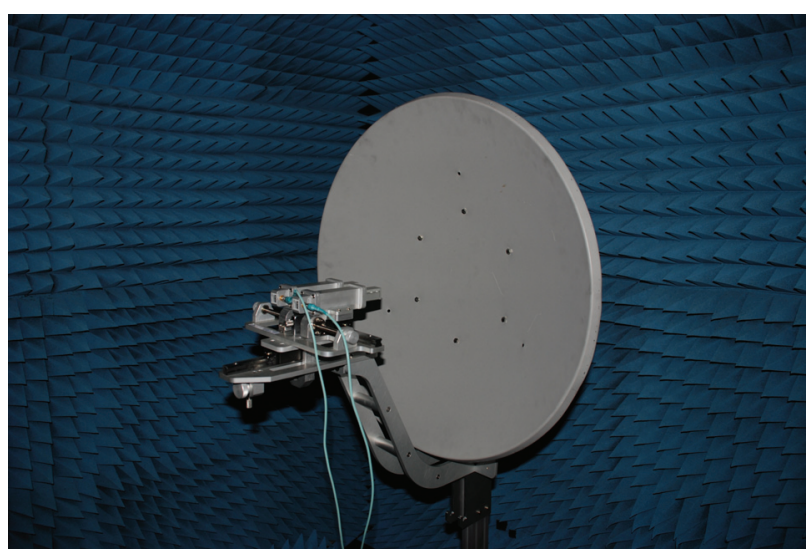

Fig. 2. Antenna under test for near-field antenna measurement

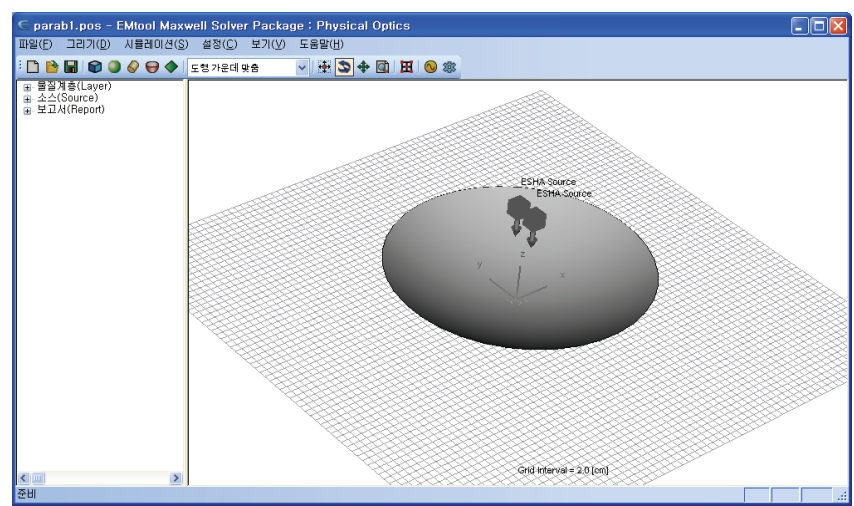

Fig. 3. Physical optics (PO) simulation setup
Our measurement is compared with the physical optics (PO) simulation illustrated in Fig. 3. The PO simulation is well suited for a high gain antenna such as a reflector antenna shown in Fig. 2. The PO simulation is mainly utilized to predict farfield patterns near to the mainbeam. At the sidelobe, the PO simulation is not good, due to diffractions from edges. These characteristics are clearly shown in Fig. 4. By inserting the UTD (Uniform Theory of Diffraction) contributions, the discrepancy between PO simulation and measurement for sidelobes can be minimized.

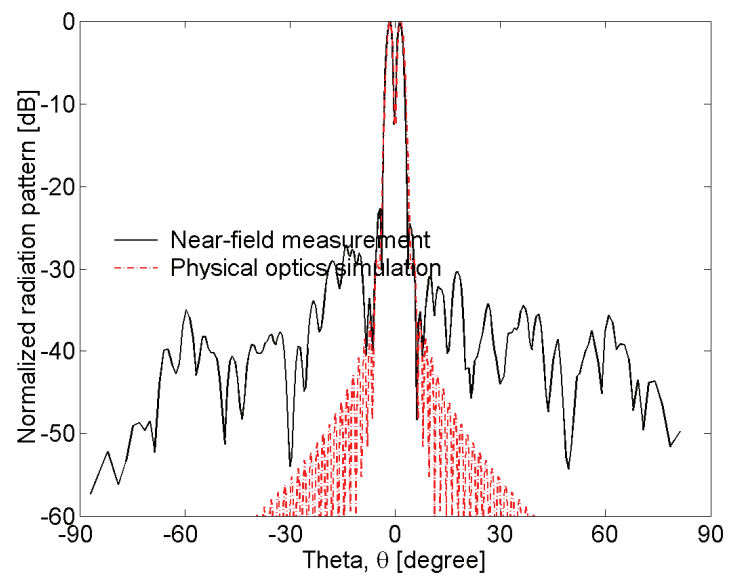

(a) Difference pattern

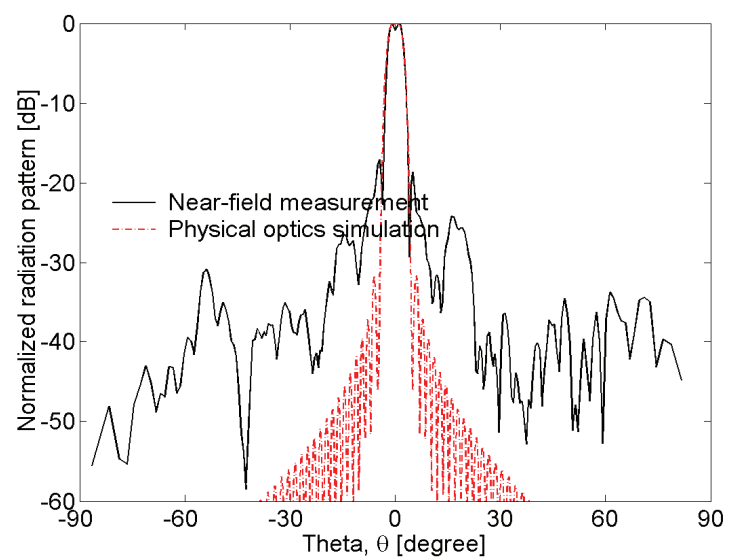

(b) Sum pattern

Fig. 4. Comparison with near-field measurement and physical optics simulation $(f=15[\mathrm{GHz}])$

Fig. 4 illustrates the behaviors of sum and difference patterns for a reflector antenna. The sum pattern is obtained with the inphase excitation of $1 \times 2$ horn array $\left(\phi_{1}=\phi_{2}\right)$ and the difference pattern does with the out of phase excitation $\left(\phi_{1}=\phi_{2}+180^{\circ}\right)$, where $\phi_{1}, \phi_{2}$ are excitation phases of the first and second horn antennas. The far-fields in Fig. 4 are compared with the near-field antenna measurement and the PO simulation. Near to the mainbeam $\left(|\theta|<5^{\circ}\right)$, the far-field results agree very well. These characteristics indicate that our formulations, (7) and (10), are correct and can be used to predict the far-field of a high gain antenna. 


\section{CONCLUSIONS}

A planar near-field antenna measurement method is proposed using symplectic relation and reaction concept. Our formulations are verified with near-field antenna measurement and physical optics simulation. Our generalized probe compensation equation can be extended to circular cylindrical and spherical surfaces as well.

\section{REFERENCES}

[1] S.-S. Oh, J.-M. Kim, and J.-H. Yun, “Angle $\phi$-variation Fresnel-zone measurement method using far-field antenna measurement system," International URSI Commission B - Electromagnetic Theory Symposium, July 2007.

[2] W.M. Leach Jr. and D.T. Paris, "Probe compensated nearfield measurements on a cylinder," IEEE Trans. Antennas Propagat., vol. 21, no. 4, July 1973, pp. 435-445.

[3] D.T. Paris, W.M. Leach Jr., and E.B. Joy, "Basic theory of probe-compensated near-field measurements," IEEE Trans. Antennas Propagat., vol. 26, no. 3, May 1978, pp. 373-379.

[4] D.M. Kerns, Plane-Wave Scattering-Matrix Theory of Antennas and Antenna-Antenna Interactions, Nat. Bur. Stand. Monograph 162, June 1981.

[5] Y.H. Cho, "The enhancement of antenna near-field measurements using near-field to far-field transform algorithms based on the Lorentz reciprocity theorem," Journal of the Korea Contents Association (in Korean), vol. 6, no. 2, Feb. 2006, pp. 51-58.

[6] Y.H. Cho, "A probe compensation equation using symplectic form," International Conference on Convergence Content, vol. 7, no. 2, Dec. 2009, pp. 597599.

[7] V.H. Rumsey, "Reaction concept in electromagnetic theory," Phys. Rev., vol. 94, no. 6, June 1954, pp. 14831491.

[8] H.-J. Li, T.-Y. Liu, and J.-L. Leou, "Antenna measurements in the presence of multipath waves," Prog. in Electromag. Research, vol. 30, 2001, pp.157-178.

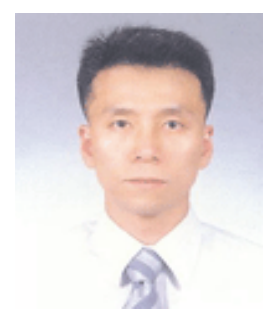

\section{Yong-Heui Cho}

He was born in Daegu, Korea, in 1972. $\mathrm{He}$ received the $\mathrm{B}$. $\mathrm{S}$. degree in Electronics Engineering from the Kyungpook National University, Daegu, Korea, in 1998, the M. S. and Ph. D. degrees in Electrical Engineering from the Korea Advanced Institute of Science and Technology (KAIST), Daejeon, Korea, in 2000 and 2002, respectively. From 2002 to 2003, he was a Senior Research Staff with the Electronics and Telecommunications Research Institute (ETRI), Daejeon, Korea. In 2003, he joined the School of Information and Communication Engineering, Mokwon University, Daejeon, Korea, where he is currently an Associate Professor. His research interests include dispersion characteristics of waveguides and electromagnetic wave scattering. 